\title{
Comparison of radiosurgery and conventional surgery for the treatment of glomus jugulare tumors
}

\author{
Oren N. GotTFried, M.D., JAMES K. LiU, M.D., \\ and William T. Couldwell, M.D., Ph.D. \\ Department of Neurosurgery, University of Utah School of Medicine, Salt Lake City, Utah
}

\begin{abstract}
Object. The optimal management of glomus jugulare tumors remains controversial. Available treatments were once associated with poor outcomes and significant complication rates. Advances in skull base surgery and the delivery of radiation therapy by stereotactic radiosurgery have improved the results obtained using these treatment options. The authors summarize and compare the contemporary outcomes and complications for these therapies.

Methods. Papers published between 1994 and 2004 that detailed the use of radiosurgery or surgery to treat glomus jugulare tumors were reviewed. Eight radiosurgery series including 142 patients and seven surgical studies including 374 patients were evaluated for neurological outcome, change in tumor size (radiosurgery) or percent of total resection (surgery), recurrences, tumor control, need for further treatment, and complications.

The mean age at treatment for patients who underwent surgery and radiosurgery was 47.3 and 56.7 years, respectively. The mean follow-up duration was 49.2 and 39.4 months, respectively. The surgical control rate was $92.1 \%$, with $88.2 \%$ of tumors totally resected in the initial surgery. A cerebrospinal fluid leak occurred in $8.3 \%$ of patients who underwent surgery and recurrences were found in $3.1 \%$; the mortality rate was $1.3 \%$. Among patients who underwent radiosurgery, tumors diminished in $36.5 \%$, whereas $61.3 \%$ had no change in tumor size, and subjective or objective improvements occurred in $39 \%$. Despite the presence of residual tumor in $100 \%$ of radiosurgically treated patients, recurrences were found in only $2.1 \%$, the morbidity rate was $8.5 \%$, and there were no deaths.

Conclusions. Death and recurrences after these treatments are infrequent, and therefore both treatments are considered to be safe and efficacious. Although surgery is associated with higher morbidity rates, it immediately and totally eliminates the tumor. The radiosurgery results are very promising, although the incidence of late recurrence (after 10-20 years) is unknown.
\end{abstract}

\section{KEY WORDS • glomus jugulare tumor • radiosurgery • radiotherapy • skull base tumor • surgery}

Glomus jugulare tumors are rare, with an estimated incidence of one per 1.3 million population. ${ }^{33}$ These tumors are typically radiosensitive, highly vascularized lesions arising from the chief cells of the paraganglia in the adventitia of the dome of the jugular bulb. Although only 1 to $5 \%$ are malignant and exhibit metastatic spread, ${ }^{3,4,6,32}$ the more common benign tumors are often locally aggressive and are associated with compression and infiltration of adjacent bone, cranial nerves, or blood vessels, ${ }^{10}$ despite an estimated growth rate of only $0.8 \mathrm{~mm}$ per year. ${ }^{5,25}$

Early attempts to treat these benign, slow-growing lesions surgically were associated with poor local tumor control, high rates of recurrence, and significant levels of morbidity and mortality. ${ }^{24}$ In 1973 , Rosenwasser ${ }^{41}$ noted a recurrence rate of $80 \%$ and a mortality rate of $50 \%$ after surgery for paragangliomas involving the skull base. In reports published in $1973^{41}$ and $1983,{ }^{40}$ separate investigators noted that the standard treatment for glomus jugulare tumors was a radical mastoidectomy, that complete excision was seldom possible, and that radiotherapy was com-

Abbreviations used in this paper: $\mathrm{CSF}=$ cerebrospinal fluid; GKS = gamma knife surgery; ICA = internal carotid artery; LINAC = linear accelerator; $\mathrm{MR}=$ magnetic resonance. monly used for residual or unresectable disease. Since that time, advances in neuroimaging, in the understanding of the surgical anatomy related to glomus jugulare tumors, in microsurgical techniques, and in the delivery of radiation have improved treatment efficacy and safety. Today, the treatment options for patients with glomus jugulare tumors include microsurgical resection, radiosurgery, vascular embolization, conventional fractionated external-beam radiotherapy, or a combination of these modalities. The goal of any treatment is to control tumor growth and prevent further neurological compromise, and it is important select the intervention with the greatest chance of tumor control and the lowest risk of complications.

For many patients the primary treatment modality for these lesions is surgery, and it is the only treatment option that can offer immediate and complete tumor elimination. Although the goal of surgery is total resection, because of their vascularity and the involvement of critical vascular and neural anatomy in glomus jugulare tumors, the total removal of these lesions has been accompanied historically by significant morbidity, including new cranial nerve deficits and occasionally death. Nevertheless, the ability to combine various skull base approaches, along with advances in microneurosurgical techniques, anesthesia, 
postoperative care, nerve grafting, embolization, cranial nerve intraoperative monitoring, and preoperative evaluation with cerebral blood flow studies have increased the possibility of achieving a total resection with fewer complications. With current techniques, large lesions, tumors involving cranial nerves, or complex glomus jugulare tumors that were deemed inoperable in the past and were treated with radiotherapy as a primary modality are being safely and totally resected. ${ }^{1,2,19,24,35,36,53}$

Conventional radiotherapy with fractionated externalbeam radiation has been used as a primary, combined, or salvage treatment in patients who cannot undergo surgery because of advanced age or other comorbidities, more aggressive, larger, or unresectable tumors, or residual disease. Its use, however, is associated with frequent recurrences at lower radiation doses, ${ }^{17,27,39,44,45,47}$ and with significant radiation-induced complications at higher doses..$^{10,14,}$ 34,38,42,44,49 These complications include radiation-induced otitis, mastoiditis, altered taste sensations, alopecia, mucositis, and dermatitis, all of which may occur acutely. Other complications that have been reported to occur in a delayed manner have included facial nerve palsy, hearing loss, temporal bone necrosis, brain necrosis, secondary malignancy including osteosarcoma, late metastasis to bone and lungs 17 years after radiation therapy, CSF leakage from radiation-induced dural and temporal bone necrosis, and pituitary/hypothalamic insufficiency. ${ }^{10,14,16 \text {, }}$ $34,38,42,44,49$ It is important to note that the goal of radiotherapy is disease control or growth inhibition rather than tumor elimination, ${ }^{43}$ because higher doses of radiation are associated with increased complications involving the temporal bone and brainstem. ${ }^{9}$ Radiation therapy has been noted to eliminate the entire tumor in only $30 \%$ of cases. ${ }^{47}$ Interestingly, the mechanism of growth inhibition of glomus jugulare tumors by radiotherapy is not entirely understood. It is thought to work by inducing perivascular fibrosis, but many tumors continue to exhibit significant vascularity on angiographic studies obtained after radiosurgery, despite decreased growth. Furthermore, unlike the vessels, the tumor (chief) cells are radioresistant and persist after radiotherapy. ${ }^{20,29,30,42,43,46}$

Recently, reports of the use of GKS and LINAC stereotactic radiosurgery as primary and secondary treatment modalities for glomus jugulare tumors have increased. ${ }^{10-12}$, 14,15,26,28-31,37,42 Glomus jugulare tumors are well demarcated on MR images and rarely invade the brain, which makes them ideal candidates for treatment with radiosurgery because it allows a steep dose decrease at the margin. ${ }^{42}$ Compared with conventional radiotherapy, radiosurgery involves a shorter treatment time, precise stereotactic localization, and a small volume of irradiated normal tissue; thus, the incidence of complications is lower. ${ }^{14,42}$ Proponents of the use of radiosurgery as a primary modality argue that it has the potential to avoid the hospital costs and potential postoperative morbidity associated with surgery, providing a more cost-effective treatment. ${ }^{14,42}$ Radiosurgical treatment usually takes 1 day, or in rare cases 2 days, compared with 4 to 6 weeks for conventionally fractionated external-beam radiation, and with several weeks of postoperative recovery for resection. ${ }^{14}$

As more outcome data for radiosurgery become available, it is important to evaluate which treatment modality is associated with the best outcomes, fewest recurrences, and fewest complications. Various authors have compared conventional radiotherapy with surgery. $7,10,48,49,51$ Carrasco and Rosenman ${ }^{7}$ evaluated 24 series that included a total of 582 patients and found that after surgery as opposed to radiotherapy as a primary treat-ment, $6 \%$ compared with $11 \%$ of patients had residual tumors, $7 \%$ compared with $8 \%$ had tumor recurrences, and the death rate was $2.5 \%$ compared with $6 \%$. Conversely, Springate and colleagues in $1991^{48}$ reviewed the literature published between 1965 and 1988 and found that the control rate for surgery alone was $86 \%$, whereas that for radiation alone was $93 \%$. In that report, the follow-up duration for the surgery series was usually more than 7 years, but the duration of follow up for the patients treated with radiotherapy alone was not reported. To our knowledge, the outcomes of radiosurgery and surgery have not been directly compared.

Our goal in this paper was to evaluate the most recent literature to determine which modality, surgery or radiosurgery, is the most effective for tumor control and the safest. Additionally, the literature was evaluated to determine whether individual tumor and patient characteristics, including the patient's age and degree of neurological impairment and the lesion's growth rate and size make one treatment modality more appropriate than the other.

\section{LITERATURE REVIEW}

This study involved a retrospective literature review of English-language articles listed in Medline that referenced the primary treatment of glomus jugulare tumors with radiosurgery or surgery, with attention focused on the larger series and those published between 1994 and 2004. A total of 10 radiosurgery articles fit this criteria, although two of these were smaller series ${ }^{10,14}$ and their data were included in later studies. ${ }^{11,15}$ Therefore, eight radiosurgery studies comprising 142 patients were evaluated for this study. Seven surgical articles met the criteria and they included 374 patients. Every attempt was made to include only patient data from glomus jugulare tumors and to exclude data from glomus tympanicum, glomus vagale, or other paraganglia tumors. When a series included some patients with these other tumors, a notation was made. Interestingly, in radiosurgery series all patients were reported to have glomus jugulare tumors.

The following parameters were evaluated: tumor size, distribution of tumor extent according to the Fisch ${ }^{13}$ and Glasscock-Jackson ${ }^{23}$ staging systems, neurological outcome, change of tumor size for radiosurgery/percentage with total resections for surgery, recurrences (radiographically or clinically confirmed), tumor control, need for further treatment, and complications.

\section{Classification Systems}

Series in which the Fisch or Glasscock-Jackson classification of the tumors were noted are listed in Table 1 for comparison. The Fisch ${ }^{13}$ system classifies five types of tumors: Class A lesions are limited to the middle ear cleft, Class B tumors are limited to the tympanomastoid area without infralabyrinthine compartment involvement, Class $\mathrm{C}$ are tumors involving the infralabyrinthine com- 
TABLE 1

Two classification systems for glomus jugulare tumors treated with radiosurgery or surgery*

\begin{tabular}{lll}
\hline \hline & \multicolumn{2}{c}{ No. of Patients/Classification } \\
\cline { 2 - 3 } \multicolumn{1}{c}{ Authors \& Year } & \multicolumn{1}{c}{ Fisch } & Glasscock-Jackson \\
\hline radiosurgery & \multicolumn{1}{c}{} \\
Eustacchio, et al., 1999 & 3/C, 5/D1, 2/D2 & 3/II, 4/III, 3/IV \\
Liscak, et al., 1999 & 36/B or C, 27/C, 17/D & NA \\
Jordan, et al., 2000 & 3/B, 3/C1, 1/D1, 1/? & NA \\
Saringer, et al., 2001 & 8/De1, 1/De2, 4/Di1 & NA \\
Eustacchio, et al., 2002 & 4/C2, 12/D1, 3/D2 & 7/II, 6/III, 6/IV \\
Maarouf, et al., 2003 & 1/C, 11/D1 or D2 & NA \\
surgery & & \\
Green, et al., 1994 & 24/B, 13/C, 15/D & NA \\
Patel, et al., 1994 & 1/B, 11/C or D & 4/II, 1/III, 7/IV \\
Whitfield, et al., 1996 & 4/B, 5/C, 6/D & NA \\
Jackson, et al., 2001 & NA & 27/I, 26/II, 44/III, \\
& & 29/IV \\
Pareschi, et al., 2003 & 25/C, 8/D1, 4/D2 & NA \\
\hline
\end{tabular}

* Not all studies provided this information. See Classification Systems for explanation of designations. Abbreviations: De1 and De2 = extradural Class D1 and D2; Di1 = intradural Class D1; NA = not available; ? = tumor not classified.

partment of the temporal bone and extending into the petrous apex, Class D1 are tumors with an intracranial extension less than $2 \mathrm{~cm}$ in diameter, and Class D2 are tumors with greater than 2-cm-diameter intracranial extension. Variants of this classification were used in some of the series reviewed in this paper. For example, "Di" refers to tumors with intradural extension, whereas "De" refers to those with extradural extension. For the GlasscockJackson classification, Grade I refers to a small tumor involving the jugular bulb, middle ear, and mastoid; Grade II is a tumor extending under the internal auditory canal; Grade III is one extending into the petrous apex; and Grade IV is one extending beyond the petrous apex and into the clivus or infratemporal fossa. Grades II, III, or IV may exhibit intracranial extension.

\section{RESULTS}

\section{Radiosurgery Series}

Demographic Data and Prevalence of GKS Compared With LINAC Therapy. Of the eight radiosurgery series evaluated (Table 2), stereotactic GKS was used in five $(62.5 \%)$ and a frame-based LINAC system was used in three $(37.5 \%) .^{10-12,14,15,26,28,30,31,42}$ In one of the studies in which LINAC was used primarily, four patients were treated with the CyberKnife. ${ }^{28}$ A total of 143 patients who were treated with radiosurgery were described. All patients received their treatments between 1990 and 2001. Sixty-nine (48\%) of 143 received radiosurgery as a primary treatment and $74(52 \%)$ had undergone other therapies prior to radiosurgery. Across all of these studies, the mean age at treatment was 56.7 years (range 44-63.5 years) and the mean duration of follow up was 39.4 months (range 20-86.4 months).

The tumor size after treatment was reported for $137 \mathrm{pa}-$ tients. Tumors were evaluated after radiosurgery by using axial, coronal, and sagittal MR images to measure the largest dimensions of each lesion in the $\mathrm{x}, \mathrm{y}$, and $\mathrm{z}$ axes. Any change in any dimension was scored as tumor growth or reduction. After treatment with stereotactic radiosurgery, at the latest follow-up review, the tumor size had decreased in $50(36.5 \%)$ of 137 lesions, and there was no change in size in $84(61.3 \%)$ of 137 . There was no case of total elimination of the tumor. The timing of the first observation of a decrease in tumor volume was documented in only one study, ${ }^{30}$ and the percentage of tumor shrinkage was reported in only one study. ${ }^{31} \mathrm{~A}$ decrease in tumor volume was observed as early as 6 months after radiosurgery and the median time reported was 20 months. ${ }^{30}$ Maarouf and colleagues reported tumor shrinkage that ranged from 25 to $74 \%$ compared with the volume noted on preradiosurgery scans in eight patients. Three patients $(2.2 \%)$ had new tumor growth after radiosurgery; this growth was observed at 6,40 , and 84 months. ${ }^{11,12}$ The cases of recurrence are discussed later.

Neurological Outcome. The neurological outcome was reported for 141 patients; it was determined by an objective change in patients' physical examination when this information was available, or if the information was not provided, it was based on changes in patients' symptoms. An improvement was noted if a patient had one or more findings of resolution or decreased intensity of the following symptoms: tinnitus, vertigo, headaches, or hearing loss. If there was partial recovery of a neurological deficit or cranial nerve function with improvements in speech, swallowing, voice quality, or shoulder strength, this was also considered to be a positive outcome.

In $82(58.2 \%)$ of 141 patients, results of the neurological examination remained stable, 55 (39\%) of 141 showed improvement, and four $(2.8 \%)$ of 141 had permanent worsening after radiosurgery according to their neurological examination. One of the four patients with permanent worsening of symptoms was later found to have metastatic disease. ${ }^{12}$ Improvements in hearing, tinnitus, or vertigo were first noticed by some patients at 1 to 6 months, and most patients who noted improvement realized it by 12 months. ${ }^{26,30}$

Complications. Overall, neurological complications occurred in $12(8.5 \%)$ of 141 patients, although nine of these cases $(6.4 \%)$ were transient. In the report by Saringer and colleagues, ${ }^{42}$ two patients experienced transient facial or glossopharyngeal neuropathies due to delayed radiation injury, one at 1 month and the other at 1 year after radiosurgery. Both conditions completely resolved within 6 months after treatment with steroid drugs. In the aforementioned study, the target volume and the administered dose were not associated with the incidence of cranial neuropathies. One patient in the study by Jordan, et al., ${ }^{26}$ experienced immediate onset of severe vertigo after radiosurgery that resolved after 11 months. Two patients suffered inflammatory complications of the inner ear. ${ }^{30}$ Four other patients experienced transient worsening of their preexisting cranial nerve deficits, tinnitus, or vertigo after radiosurgery that resolved without treatment. ${ }^{12,15,28}$

The rate of permanent deficits associated with radiosurgery was three $(2.1 \%)$ of 141 patients. ${ }^{12,30,31}$ Liscak and colleagues ${ }^{30}$ described two patients with permanent worsening, in one of whom facial nerve function deteriorated to House-Brackmann Grade II and hearing loss pro- 
TABLE 2

Literature review of patient information and outcomes after radiosurgical treatment of glomus jugulare tumors*

\begin{tabular}{|c|c|c|c|c|c|c|c|c|c|c|c|}
\hline Authors \& Year & Treatment & $\begin{array}{c}\text { Mean } \\
\text { Patient } \\
\text { Age (yrs) }\end{array}$ & $\begin{array}{l}\mathrm{FU} \\
(\mathrm{mos})\end{array}$ & $\begin{array}{l}\text { Tumor } \\
\text { Vol } \\
\left(\mathrm{cm}^{3}\right)\end{array}$ & $\begin{array}{l}\text { No. of } \\
\text { Patients }\end{array}$ & $\begin{array}{l}\text { Long-Term } \\
\text { Neurological } \\
\text { Outcome }\end{array}$ & $\begin{array}{c}\% \\
\text { Clinically } \\
\text { Improved }\end{array}$ & $\begin{array}{l}\text { Change in } \\
\text { Tumor } \\
\text { Size }\end{array}$ & $\begin{array}{l}\% \text { w/ } \\
\text { Reduced } \\
\text { Tumor } \\
\text { Size }\end{array}$ & $\begin{array}{l}\text { Recur- } \\
\text { rence } \\
(\%)\end{array}$ & Complications \\
\hline $\begin{array}{l}\text { Eustacchio, et al., } \\
1999 \dagger\end{array}$ & GKS & 52.4 & 37.6 & 6.4 & $10(4 \mathrm{P}, 6 \mathrm{~S})$ & $5 \mathrm{U}, 5 \mathrm{I}$ & 50 & $6 \mathrm{U}, 4 \mathrm{r}$ & 40 & 0 & 0 \\
\hline Liscak, et al., 1999 & GKS & 54.0 & 24.0 & 5.7 & $52(24 \mathrm{P}, 28 \mathrm{~S})$ & $35 \mathrm{U}, 15 \mathrm{I}, 2 \mathrm{~W}$ & 29 & $28 \mathrm{U}, 19 \mathrm{r}, 5 \mathrm{~L}$ & 40 & 0 & 3 trans, 2 perm \\
\hline $\begin{array}{l}\text { Eustacchio, et al., } \\
2002 \ddagger\end{array}$ & GKS & 56.0 & 86.4 & 5.2 & 19 (10P, 9S) & $8 \mathrm{U}, 10 \mathrm{I}, 1 \mathrm{~L}$ & 53 & 11U, 7r, 1inc & 37 & $\begin{array}{l}1 / 18 \\
(5.6)\end{array}$ & 0 \\
\hline $\begin{array}{l}\text { Feigenberg, et al., } \\
2002\end{array}$ & LINAC & 49.0 & 27.0 & 10.8 & $5(4 \mathrm{P}, 1 \mathrm{~S})$ & $2 \mathrm{U}, 2 \mathrm{I}, 1 \mathrm{~W}$ & 40 & $3 \mathrm{U}, 2 \mathrm{inc}$ & 0 & $\begin{array}{l}2 / 5 \\
(40)\end{array}$ & 1 trans \\
\hline Foote, et al., $2002 \S$ & GKS & 62.5 & 37.0 & 10.4 & $25(12 \mathrm{P}, 13 \mathrm{~S})$ & $10 \mathrm{U}, 15 \mathrm{I}$ & 60 & $17 \mathrm{U}, 8 \mathrm{r}$ & 32 & 0 & 1 trans \\
\hline Lim, et al., 2003 & CK/LINAC & 44.0 & 26.0 & NA & $9(4 \mathrm{P}, 5 \mathrm{~S})$ & $9 \mathrm{U}$ & 0 & $8 \mathrm{U}, 1 \mathrm{r}$ & 11 & 0 & 1 trans \\
\hline
\end{tabular}

* $\mathrm{CK}=$ Cyberknife; $\mathrm{FU}=$ follow up; $\mathrm{I}=$ improved; inc $=$ increased $\mathrm{L}=$ lost to follow up; $\mathrm{P}=$ primary treatment; perm $=$ permanent; $\mathrm{r}=$ reduced; $\mathrm{S}=$ secondary treatment; trans = transient; $\mathrm{U}=$ unchanged; $\mathrm{W}=$ worse.

$\dagger$ Data from this study were not included in the overall review because these results were included in the larger series published by the same primary author.

\$ Series included the 10 patients from Eustacchio, et al., 1999.

$\S$ Series included the nine patients from Foote, et al., 1997.

gressed to deafness over 12 months. The other patient's facial nerve function deteriorated to Grade IV and vertigo worsened at 6 months. Maarouf and colleagues ${ }^{31}$ had one patient in whom facial weakness developed 6 months after radiosurgery. No deaths were associated with the use of radiosurgery.

Additional Therapy. In several series, patients underwent additional surgery (one patient), embolization (four patients), or further radiosurgery (five patients) after their initial radiosurgical treatment, although in these cases there was no evidence of radiographically or clinically confirmed recurrence at the time of the additional treatments, and many were treated for persistent tinnitus. ${ }^{10}$, ${ }_{11,30,31}$ After a mean follow-up duration of 39.4 months, three $(2.2 \%)$ of 137 patients with complete follow up experienced clinically or radiographically confirmed recurrences. ${ }^{11,12}$ One patient had been treated with radiosurgery as a primary modality and had evidence of new disease at the field's margin at 6 months posttreatment. ${ }^{12}$ This patient was treated with salvage radiotherapy and had no further disease progression or evidence of recurrence 21 months later. Another patient was treated with radiosurgery for recurrent disease and experienced another recurrence 40 months postradiosurgery. She was subsequently found to have metastatic disease in her lymph nodes and no further treatment was performed. ${ }^{12}$ Finally, one patient who was treated with radiosurgery after a partial resection suffered a recurrence at 84 months that was thought to be due to undertreatment stemming from the inability to identify the whole tumor on computerized tomography planning studies. ${ }^{11}$

Radiosurgery as Primary Treatment. Although 69 of 143 patients underwent radiosurgery as a primary modality, very few series provided data on these individuals separately. ${ }^{10,26}$ Typically, data were provided for all pa- tients combined, and it was impossible to decipher the outcomes and complications in those who received radiosurgery as a primary modality. In two series, reported by Eustacchio, et al. ${ }^{10}$ and Jordan, et al., ${ }^{26}$ eight patients received radiosurgery as a primary treatment; tumor size remained the same for six (75\%) and was reduced for two (25\%), and the neurological outcome improved for the other four $(50 \%)$ and remained the same for four $(50 \%)$. Feigenberg and colleagues ${ }^{12}$ described one patient who experienced a recurrence after receiving radiosurgery as a primary treatment.

Radiosurgery as Secondary Treatment. Radiosurgery was used as secondary therapy in 74 (52\%) of 143 patients. Of these patients, $60.3 \%$ underwent surgery prior to radiosurgery, $15.1 \%$ had radiation therapy, and $24.6 \%$ underwent embolization (some of them had combinations of these treatments). Foote and coworkers ${ }^{15}$ found a reduction in tumor size after radiosurgery in seven of 12 patients who had previously undergone surgery. Again, in most series the authors did not document separate data for the patients who underwent other treatments that failed prior to radiosurgery.

\section{Surgery Series}

Demographic Data and Overview of Approaches. A total of 374 patients were treated with 384 surgical procedures in the seven series we evaluated (Table 3). All patients underwent surgery during the period from 1972 to 2000. Across all of these studies, the mean age at treatment was 47.3 years (range $41.7-49$ years) and the mean duration of follow up was 49.2 months (range 37.2-58.8 months). Green, et al. ${ }^{19}$ published the only series that included previously untreated glomus jugulare tumors. Between 7.7 and $50 \%$ of patients had previously undergone surgery and between 5.5 and $18 \%$ had previously under- 
TABLE 3

Literature review of patient outcomes after surgical treatment of glomus jugulare tumors*

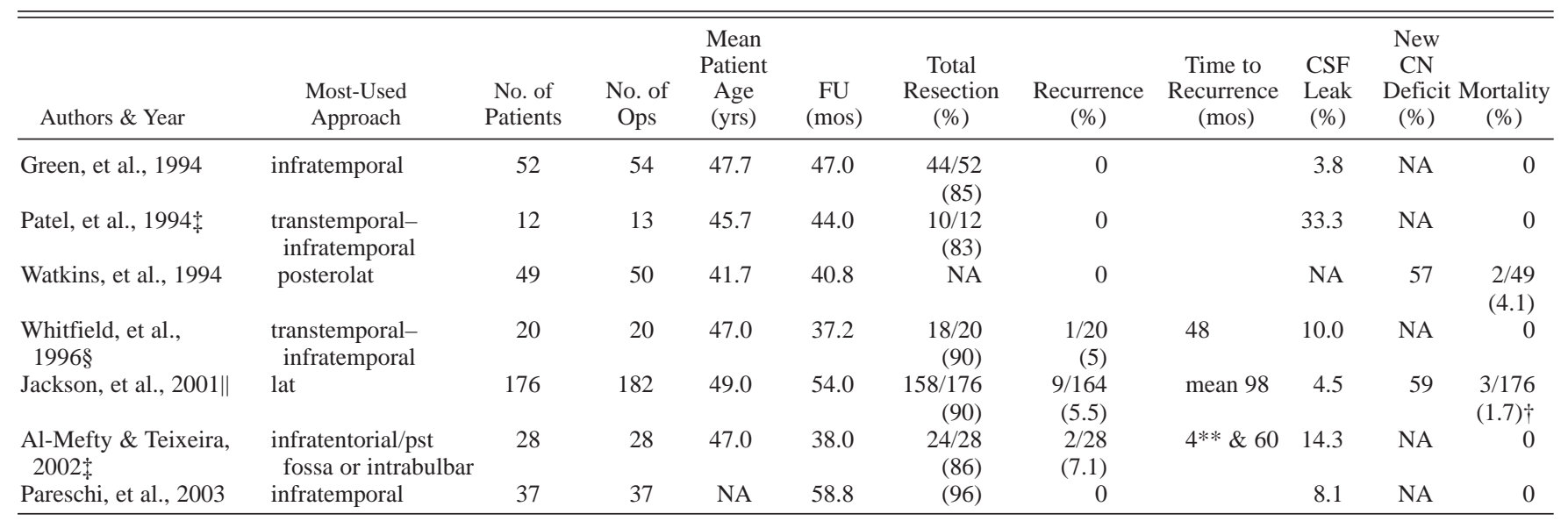

$* \mathrm{CN}=$ cranial nerve; $\mathrm{pst}=$ posterior.

$\uparrow$ Two additional patients died, but they had carotid body tumors.

¥ Series included complex glomus jugulare tumors.

$\S$ Series included five glomus vagale tumors.

|| Series included 27 glomus vagale tumors and three carotid body tumors.

** Recurrence occurred at 4 months in a patient with a malignant glomus jugulare tumor.

gone radiation treatment. ${ }^{24,35,36,52,53}$ Approximately 25\% of patients underwent preoperative embolization, ${ }^{2,19,36,53}$ but in the study by Pareschi, et al., ${ }^{35} 100 \%$ of patients had received embolization. Tumors were treated in single operation $^{2,19,24,35,52,53}$ or with staged surgery. ${ }^{35,36}$ The approaches included lateral, ${ }^{24}$ posterolateral with posterior fossa craniectomy and infratemporal fossa approach, ${ }^{35,52}$ infratemporal fossa approach (first stage) followed by petrooccipital transsigmoid approach (second stage), ${ }^{35}$ jugulopetrosectomy, ${ }^{35}$ transtemporal-infratemporal, ${ }^{36,53}$ subtemporal-infratemporal added to a transtemporal-infratemporal, ${ }^{36}$ transtemporal-infratemporal with a retrosigmoid craniectomy and/or extreme-lateral transcondylar approach, ${ }^{36}$ infratentorial and posterior fossa, ${ }^{2}$ and intrabulbar dissection. ${ }^{2}$

Series With Complex Tumors. It is important to note

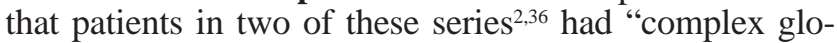
mus jugulare tumors" as described by Al-Mefty and Teixeira, which include giant tumors, multiple paragangliomas, malignancy, catecholamine secretion, previous treatment with adverse events such as sacrifice of the ICA that make surgical intervention more risky, prior radiation, or adverse effects from embolization. The rate of total resection of lesions in these series that included challenging tumors was 83 and $86 \%$.

Series Without Complex Tumors. Overall, total resection was achieved on the first attempt in $254(88.2 \%)$ of 288 surgeries, although this number excluded cases from two series that did not provide enough information. . $^{35,52,53}$ On evaluation of the series that did not include complex tumors, 85 to $96 \%$ of surgeries resulted in total resections. ${ }^{2,19,24,35,36,52,53}$ In a series by Jackson, et al., ${ }^{24}$ all subtotal resections (18 [10\%] of 182) were for Grade III or IV tumors and were planned based either on preoperative patient preference for preservation of cranial nerves or the ICA, or to perform palliative surgery in elderly patients.
Most of the subtotal resections occurred in Fisch Class D tumors, and some surgeons achieved total resections for Class C tumors in $100 \%$ of cases. ${ }^{35}$

The surgical control rate for the noncomplex tumor series ranged from 88.5 to $94.5 \% .^{19,24,36,53}$ Surgical control was defined as complete tumor elimination with no evidence of residual or recurrent disease throughout the follow-up period. For example, in the report by Green, et al. ${ }^{19}$ two of eight patients with subtotally resected tumors underwent immediate repeated surgeries to remove the residual tumors; thus, only six $(11.5 \%)$ of 52 patients remained with residual disease, and tumor control was $88.5 \%$. After repeated surgeries for residual or recurrent disease, the surgical control rate was $92 \%$.

Preservation of Cranial Nerves. Cranial nerve preservation was most easily accomplished in smaller tumors and was as high as 80 to $95 \% . .^{21,22,36}$ In one series, ${ }^{24} \mathrm{com}-$ plete removal of a glomus jugulare tumor without cranial nerve resection or a new deficit occurred in $31 \%$ of patients. In the aforementioned series, the ninth through 12th cranial nerves had to be resected in $34.6 \%$ of cases, and this was mostly because of tumor involvement in the lower cranial nerves at the pars nervosa of the jugular bulb. According to our review of the literature, one or more new cranial nerve deficits occurred in 22 to $59 \%$ of patients after surgery, ${ }^{8,18,24,52}$ whereas in another review new seventh, ninth, or 10th cranial nerve dysfunction was noted in 49 to $83 \%$ of patients. ${ }^{10}$ Jackson, et al. ${ }^{24}$ found that although it was necessary to sacrifice the seventh, ninth, and 10 th cranial nerves in 23,63 , and $59 \%$ of cases, respectively, preoperative deficits existed only in 12,21 , and $31 \%$, respectively. In several recent series new deficits were noted in the seventh cranial nerve in 4.4 to $11 \%$ of cases, in the ninth in 26 to $42 \%$, in the 10th in 13 to $28 \%$, in the 11th in 25 to $26 \%$, and in the 12th in 5 to $21 \% .{ }^{19,21,24}$

Although almost all patients experience facial weakness in the postoperative period after nerve transposition 
O. N. Gottfried, J. K. Liu, and W. T. Couldwell

or grafting, functional outcomes in the facial nerve are improving with the currently available advanced microsurgical techniques, use of facial nerve monitoring, and limiting of nerve transposition when possible. ${ }^{19,36,53}$ For example, Green and coworkers ${ }^{19}$ noted recovery of function to House Grade I or II in 95\% of patients on longterm follow-up review, and Whitfield and colleagues ${ }^{53}$ noted that only $25 \%$ of patients had facial nerve dysfunction on follow up. Patel and coworkers ${ }^{36}$ were able to maintain sensorineural hearing in $11(91.7 \%)$ of 12 patients.

Complications. The incidences of other morbidities or postoperative complications are listed in Table 4. ${ }^{2,19,24,35 \text {, }}$ 36,52,53 The most common complications were CSF leakage $(8.3 \%)$, aspiration $(5.5 \%)$, wound infection/ischemia $(5.5 \%)$, pneumonia $(2.3 \%)$, and meningitis $(2.1 \%)$. None of these complications resulted in long-term deficits or required rehospitalization after initial discharge.

Additional Therapy. Adjunctive procedures were required in some patients after surgery and included vocal cord injection (10-25\%), thyroplasty (8-10\%), eyelid weights (percentage not available), cricopharyngeal myotomy (4\%), tracheostomy (0-5.4\%), gastrostomy (4-5\%), lateral tarsorrhaphy (20\%), and nasogastric tube feeding (8-30\%). ${ }^{19,35,53}$ Whitfield, et al., ${ }^{53}$ noted that patients in their series who had lower cranial nerve deficits did well after undergoing these adjunctive procedures; for example, five patients with dysphonia attained a complete resolution after vocal cord injection or thyroplasty. Patel and coworkers ${ }^{36}$ treated 10 patients who had swallowing difficulties postoperatively, and symptoms resolved in nine of them without the need for a feeding tube. Several authors noted that patients with preoperative ninth and 10th cranial nerve deficits had an earlier and greater improvement in swallowing after surgery than patients without preoperative deficits, ${ }^{8,36}$ and in another paper it was reported that morbidity related to these cranial nerve deficits was greatly decreased by aggressive pre- and postoperative speech and swallowing therapy. ${ }^{54}$ Another group noted a decrease in the need for tracheostomy and gastrostomy tubes after surgery. ${ }^{54}$

As mentioned earlier, the overall incidence of CSF leakage was $8.3 \%$, and was a significant complication associated with surgery for glomus jugulare tumors. The incidence of CSF leakage ranged from 3.8 to $10 \%$ in most series, ${ }^{19,24,35,52,53}$ although in the two series with more than $50 \%$ of repeated surgeries the incidence was 14.3 to $33.3 \%$. 2,36 The majority of CSF leaks resolved without the need for repeated surgical closure after being treated with conservative management or a lumbar drain. ${ }^{19,35,36}$ In the study by Whitfield and coworkers, ${ }^{53}$ two patients had to undergo additional surgery to repair CSF leaks. In one series, intracranial, transdural tumor extension was present in 36\% of cases, and CSF leaks were encountered in 64\% of procedures. ${ }^{24}$ The risk of CSF leakage was decreased with improvements in dural closure including reconstruction with a vascularized local, regional, or free flap, such as a temporoparietal fascia flap. ${ }^{24,36}$ With a good dural closure, in one series a decrease in the incidence of CSF leakage from 28 to $4.5 \%$ was found. ${ }^{24}$ Other practitioners lowered the risk of CSF leakage for large intradural tumors by performing staged procedures. ${ }^{35}$
TABLE 4

Complications after 384 surgeries for glomus jugulare tumor

\begin{tabular}{lc}
\hline \hline \multicolumn{1}{c}{ Complication } & No. of Patients $(\%)$ \\
\hline CSF leak & $32(8.3)$ \\
aspiration & $21(5.5)$ \\
wound infection/ischemia & $21(5.5)$ \\
pneumonia & $9(2.3)$ \\
meningitis & $8(2.1)$ \\
ileus & $6(1.6)$ \\
cerebral hemorrhage & $6(1.6)$ \\
stroke & $6(1.6)$ \\
external ear infection & $5(1.3)$ \\
tympanic membrane perforation & $4(1.0)$ \\
pulmonary embolus & $3(0.8)$ \\
worsened ataxia & $3(0.8)$ \\
pneumothorax & $3(0.8)$ \\
osteomyelitis of temporal bone & $2(0.5)$ \\
hydrocephalus & $2(0.5)$ \\
seroma & $2(0.5)$ \\
deep vein thrombosis & $1(0.3)$ \\
myocardial infarction & $1(0.3)$ \\
tracheitis & $1(0.3)$ \\
\hline
\end{tabular}

Overall, there were five deaths related to surgery in 374 patients, for a total mortality rate of $1.3 \%$. The mortality rate after surgery ranged from 0 to $4.1 \%$., $19,24,35,36,52,53$ Deaths occurred from stroke caused by ICA injury (two patients), intracerebral hemorrhage (one patient), and pulmonary embolus (two patients). ${ }^{24,52}$ The incidence of stroke, hemorrhage, or pulmonary embolus in the literature was low, at 1.6, 1.6, and $0.8 \%$, respectively (Table 4).

Tumor Recurrence. Recurrence of glomus jugulare tumors after surgery was noted in 12 (3.3\%) of 360 cases; the range in these series was 0 to $5.5 \%, 2,19,24,35,36,52,53$ and the mean time to recurrence was 82.8 months. ${ }^{2,24,53}$ For benign glomus jugulare tumors, recurrences were reported at a range of 25 to 273 months. ${ }^{2,24,53} \mathrm{Al}$-Mefty and Teixeira ${ }^{2}$ described a patient in whom they achieved total resection of a very extensive and malignant glomus jugulare tumor after failure of radiation therapy, and the tumor recurred 4 months later. In the report by Jackson, et al., ${ }^{24}$ nine of 18 subtotally resected tumors recurred; five of these cases were treated with total resection, two were observed, one was treated with radiotherapy, and one was left untreated.

Pareschi and coworkers ${ }^{35}$ noted that $84 \%$ of their patients were sent home within 14 days of surgery, and in another two studies the mean hospital stay was 10 days. ${ }^{19,54}$ Green and colleagues ${ }^{19}$ noted that $85 \%$ of patients were able to resume fully all preoperative activities and to take care of themselves. In another study it was found that all patients who were able to live independently before surgery continued to be independent. ${ }^{36}$ In another series, 70\% of patients subjectively noted that they had an excellent functional outcome..$^{53}$

\section{DISCUSSION}

Since the original description of glomus jugulare tumors in the 1940s, there has been considerable controversy regarding the best management of this disease. In the past, observation, surgery, and radiotherapy were all used as primary treatments for these lesions. One factor that makes selecting the appropriate treatment difficult is the 
variable growth rate and clinical course of these tumors. Although the majority are benign and slow-growing lesions, they are locally invasive and, if left untreated, they have the potential to progress to cause considerable cranial nerve and brainstem injury in some patients. In the past, the main arguments against surgical intervention were the high percentage of incomplete resections and, more importantly, the high rates of morbidity and mortality. Surgeons, however, argued that this modality was the only one with which they could obtain a complete removal of the tumor. Radiotherapy was criticized for the high exposure of normal neural tissue and bone to radiation, resulting in considerable radiation-induced complications. Additional criticisms included the delay in the treatment's effect and the fact that residual tumor remained after a successful intervention.

Our review demonstrates that the treatment of glomus jugulare tumors is now safer and more efficacious than it has ever been. This improvement is most likely due to considerable advances in surgery and in the delivery of radiation by using stereotactic radiosurgery. In the surgical literature we reviewed, we found that $88.2 \%$ of tumors were totally resected on the first surgery, with a surgical control rate of $92.1 \%$. Cranial nerve preservation improved, the incidence of CSF leakage decreased, recurrences were noted in only $3.1 \%$ of patients, and the mortality rate was $1.3 \%$.

Among radiosurgical series, in $36.5 \%$ of patients the tumors decreased in size, $61.3 \%$ of tumors stayed the same size, all patients $(100 \%)$ had residual tumors, $39 \%$ of patients had subjective or objective improvements, recurrences were found in $2.1 \%$, the morbidity rate was $8.5 \%$, and there were no deaths. Interestingly, the question of whether one defines treatment success as a cure by total elimination of tumor (surgery definition) or growth inhibition (radiosurgery definition) remains, and makes it difficult to compare these modalities.

The radiosurgery and surgery groups in this review were similar. The mean patient age in the surgery series was 47.3 years, compared with 56.7 years in the radiosurgery series. The patients who underwent surgery were followed for 49.2 months and the patients treated with radiosurgery were followed for 39.4 months. It is significant to note that, whereas all of the radiosurgery patients were treated in 1990 or later, many of the surgery patients were treated in the 1970s and 1980s, and therefore the overall results for the patients who were more recently treated with surgery may be even better than reported in this review. It is difficult to make any comparison or to draw any conclusion about the sizes or classifications of the tumors in these two groups because of the variability in the way the data were reported. Additionally, the current classification systems do not account for various important surgical factors such as brainstem compression, degree of vascular involvement, or large tumor size. ${ }^{36}$

Nonetheless, the data support the fact that recurrence after either treatment is very low and is similar at 3 or 4 years posttreatment, regardless of how one defines control. It will be interesting to analyze more long-term studies to see how recurrence rates compare at 10 and 20 years, because both surgery and radiosurgery now have such promising results. Many authors argue that the true test of a cure cannot be confirmed until observation has continued for at least 10 or 20 years. Liscak, et al. ${ }^{30}$ warn that a 5-year follow-up period cannot be considered adequate to judge the success of the treatment. It is well known that tumor cells persist after radiation therapy. Therefore, it will be very important to see whether patients who undergo radiosurgery, who all have persistent tumors despite growth stabilization or reduction, experience increased recurrences at 10 and 20 years compared with those treated surgically. For example, in the radiosurgery study with the longest follow-up duration (mean 7 years) ${ }^{11}$ there was a recurrence at 7 years. In a study in which patients were treated exclusively with radiation therapy, ${ }^{39}$ the authors found that the tumor control rate was $90 \%$ at 10 years, but $73 \%$ at 25 years.

No deaths were associated with radiosurgery and the mortality rate secondary to surgery was a little more than $1 \%$. The biggest difference between surgery and radiosurgery is the rate of morbidity. Although it is easy to compute the morbidity associated with radiosurgery $(8.5 \%)$, it is difficult to establish the short- and long-term morbidity in surgically treated patients. These patients are faced with many possible complications (Table 4). Furthermore, although it is safe to say that more than $8.5 \%$ of the surgical group will have a new cranial nerve deficit after surgery for larger tumors, the impact of this new deficit on a patient's long-term outcome is difficult to interpret in the surgical papers. Long-term cranial nerve functional outcomes in patients with new deficits (aside from those involving the facial nerve) were not commonly reported in the surgical series. The surgical series did report that when cranial nerve dysfunction occurred, the use of adjunctive procedures and rehabilitation resulted in lower associated morbidity from this new deficit, and that many patients eventually improved to preoperative status. Proponents of radiosurgery as a primary modality argue that it has the potential to avoid the hospital costs and potential postoperative morbidity associated with surgery, providing a more cost-effective treatment. ${ }^{14,42}$ As mentioned earlier, radiosurgical treatment usually takes 1 day, 2 days in rare cases, compared with a mean 10-day hospital stay after surgery and possibly more if rehabilitation is needed.

Surgery remains the treatment of choice in an otherwise healthy patient who desires the immediate cure of disease provided by a total resection. Al-Mefty and Teixeira ${ }^{2}$ have shown that even the most complex glomus jugulare tumors can be totally resected with minimal complications. Other strong indications for surgery are severe cranial nerve palsies, tumors that are too large for radiosurgery, lesions that cause vascular insufficiency due to major arterial encasement, or tumors that have significant intracranial extension and are life threatening. ${ }^{50}$ Obviously, resection is more difficult in cases with brainstem compression or major encasement of arteries by tumor, ${ }^{36}$ and if a complete resection cannot be guaranteed or if there is a high risk of neurological deficits or morbidity with a radical resection, then a subtotal resection can be performed to debulk the tumor and prevent further neurological compromise. After a subtotal resection, stereotactic radiosurgery is a safe and effective option to treat residual tumor. Finally, elderly patients or those with significant comorbidities may elect to undergo radiosurgery as a primary treatment modality. ${ }^{10}$ The same guideline applies to cases of recurrent glomus jugulare tumors. 


\section{Acknowledgment}

We acknowledge the superb editorial assistance of Ms. Kristin Kraus.

\section{References}

1. Al-Mefty O, Fox JL, Rifai A, et al: A combined infratemporal and posterior fossa approach for the removal of giant glomus tumors and chondrosarcomas. Surg Neurol 28:423-431, 1987

2. Al-Mefty O, Teixeira A: Complex tumors of the glomus jugulare: criteria, treatment, and outcome. J Neurosurg 97: 1356-1366, 2002

3. Bojrab DI, Bhansali SA, Glasscock ME III: Metastatic glomus jugulare: long-term followup. Otolaryngol Head Neck Surg 104:261-264, 1991

4. Brewis C, Bottrill ID, Wharton SB, et al: Metastases from glomus jugulare tumors. J Laryngol Otol 114:17-23, 2000

5. Brown JS: Glomus jugulare tumors. Methods and difficulties of diagnosis and surgical treatment. Laryngoscope 77:26-67, 1967

6. Brown JS: Glomus jugulare tumors revisited: a ten-year statistical follow-up of 231 cases. Laryngoscope 95:284-288, 1985

7. Carrasco V, Rosenman J: Radiation therapy of glomus jugulare tumors. Laryngoscope 103 (Suppl 60):23-27, 1993

8. Cece JA, Lawson W, Biller HF, et al: Complications in the management of large glomus jugulare tumors. Laryngoscope 97:152-157, 1987

9. Cole DJ: Glomus jugulare tumours seen in Oxford 1960-1984. Clin Radiol 39:83-86, 1988

10. Eustacchio S, Leber K, Trummer M, et al: Gamma knife radiosurgery for glomus jugulare tumours. Acta Neurochir 141: 811-818, 1999

11. Eustacchio S, Trummer M, Unger F, et al: The role of Gamma Knife radiosurgery in the management of glomus jugular tumours. Acta Neurochir Suppl 84:91-97, 2002

12. Feigenberg SJ, Mendenhall WM, Hinerman RW, et al: Radiosurgery for paraganglioma of the temporal bone. Head Neck 24:384-389, 2002

13. Fisch U: Infratemporal fossa approach for glomus tumors of the temporal bone. Ann Otol Rhinol Laryngol 91:474-479, 1982

14. Foote RL, Coffey RJ, Gorman DA, et al: Stereotactic radiosurgery for glomus jugulare tumors: a preliminary report. Int J Radiat Oncol Biol Phys 38:491-495, 1997

15. Foote RL, Pollock BE, Gorman DA, et al: Glomus jugulare tumor: tumor control and complications after stereotactic radiosurgery. Head Neck 24:332-339, 2002

16. Gabriel EM, Sampson JH, Dodd LG, et al: Glomus jugulare tumor metastatic to the sacrum after high-dose radiation therapy: case report. Neurosurgery 37:1001-1005, 1995

17. Gibbin KP, Henk JM: Glomus jugulare tumours in South Wales-a twenty-year review. Clin Radiol 29:607-609, 1978

18. Gjuric M, Rudiger Wolf S, Wigand ME, et al: Cranial nerve and hearing function after combined-approach surgery for glomus jugulare tumors. Ann Otol Rhinol Laryngol 105:949-954, 1996

19. Green JD Jr, Brackmann DE, Nguyen CD, et al: Surgical management of previously untreated glomus jugulare tumors. Laryngoscope 104:917-921, 1994

20. Hawthorne MR, Makek MS, Harris JP, et al: The histopathological and clinical features of irradiated and nonirradiated temporal paragangliomas. Laryngoscope 98:325-331, 1988

21. Jackson CG, Cueva RA, Thedinger BA, et al: Conservation surgery for glomus jugulare tumors: the value of early diagnosis. Laryngoscope 100:1031-1036, 1990

22. Jackson CG, Cueva RA, Thedinger BA, et al: Cranial nerve preservation in lesions of the jugular fossa. Otolaryngol Head Neck Surg 105:687-693, 1991

23. Jackson CG, Glasscock ME III, Harris PF: Glomus tumors. Di- agnosis, classification, and management of large lesions. Arch Otolaryngol 108:401-410, 1982

24. Jackson CG, McGrew BM, Forest JA, et al: Lateral skull base surgery for glomus tumors: long-term control. Otol Neurotol 22:377-382, 2001

25. Jansen JC, van den Berg R, Kuiper A, et al: Estimation of growth rate in patients with head and neck paragangliomas influences the treatment proposal. Cancer 88:2811-2816, 2000

26. Jordan JA, Roland PS, McManus C, et al: Stereotactic radiosurgery for glomus jugulare tumors. Laryngoscope 110:35-38, 2000

27. Kim JA, Elkon D, Lim ML, et al: Optimum dose of radiotherapy for chemodectomas of the middle ear. Int J Radiat Oncol Biol Phys 6:815-819, 1980

28. Lim M, Gibbs IC, Adler JR Jr, et al: The efficacy of linear accelerator stereotactic radiosurgery in treating glomus jugulare tumors. Technol Cancer Res Treat 2:261-265, 2003

29. Liscak R, Vladyka V, Simonova G, et al: Leksell gamma knife radiosurgery of the tumor glomus jugulare and tympanicum. Stereotact Funct Neurosurg 70 (Suppl 1):152-160, 1998

30. Liscak R, Vladyka V, Wowra B, et al: Gamma knife radiosurgery of the glomus jugulare tumour-early multicentre experience. Acta Neurochir 141:1141-1146, 1999

31. Maarouf M, Voges J, Landwehr P, et al: Stereotactic linear accelerater-based radiosurgery for the treatment of patients with glomus jugulare tumors. Cancer 97: 1093-1098, 2003

32. Manolidis S, Shohet JA, Jackson CG, et al: Malignant glomus tumors. Laryngoscope 109:30-34, 1999

33. Moffat DA, Hardy DG: Surgical management of large glomus jugulare tumours: infra-and trans-temporal approach. J Laryngol Otol 103:1167-1180, 1989

34. Mumber MP, Greven KM: Control of advanced chemodectomas of the head and neck with irradiation. Am J Clin Oncol 18:389-391, 1995

35. Pareschi RR, Righini S, Destito D, et al: Surgery of glomus jugulare tumors. Skull Base 13:149-158, 2003

36. Patel SJ, Sekhar LN, Cass SP, et al: Combined approaches for resection of extensive glomus jugulare tumors. A review of 12 cases. J Neurosurg 80:1026-1038, 1994

37. Pendl G, Schrottner O, Friehs GM, et al: Radiosurgery with the first Austrian cobalt-60 Gamma-unit. A one year experience. Acta Neurochir 127:170-179, 1994

38. Pluta RM, Ram Z, Patronas NJ, et al: Long-term effects of radiation therapy for a catecholamine-producing glomus jugulare tumor. Case report. J Neurosurg 80:1091-1094, 1994

39. Powell S, Peters N, Harmer C: Chemodectoma of the head and neck: results of treatment in 84 patients. Int J Radiat Oncol Biol Phys 22:919-924, 1992

40. Reddy EK, Mansfield CM, Hartman GV: Chemodectoma of glomus jugulare. Cancer 52:337-340, 1983

41. Rosenwasser H: Long-term results of therapy of glomus jugulare tumors. Arch Otolaryngol 97:49-54, 1973

42. Saringer W, Khayal H, Ertl A, et al: Efficiency of gamma knife radiosurgery in the treatment of glomus jugulare tumors. Minim Invasive Neurosurg 44:141-146, 2001

43. Schwaber MK, Gussack GS, Kirkpatrick W: The role of radiation therapy in the management of catecholamine-secreting glomus tumors. Otolaryngol Head Neck Surg 98:150-154, 1988

44. Sharma PD, Johnson AP, Whitton AC: Radiotherapy for jugulo-tympanic paragangliomas (glomus jugulare tumours). J Laryngol Otol 98:621-629, 1984

45. Simko TG, Griffin TW, Gerdes AJ, et al: The role of radiation therapy in the treatment of glomus jugulare tumors. Cancer 42: 104-106, 1978

46. Spector GJ, Compagno J, Perez CA, et al: Glomus jugulare tumors: effects of radiotherapy. Cancer 35:1316-1321, 1975

47. Spector GJ, Fierstein J, Ogura JH: A comparison of therapeutic modalities of glomus tumors in the temporal bone. Laryngoscope 86:690-696, 1976 


\section{Comparison of treatments for glomus jugulare tumors}

48. Springate SC, Haraf D, Weichselbaum RR: Temporal bone chemodectomas-comparing surgery and radiation therapy. Oncology 5:131-137, 140, 143, 1991

49. Springate SC, Weichselbaum RR: Radiation or surgery for chemodectoma of the temporal bone: a review of local control and complications. Head Neck 12:303-307, 1990

50. van der Mey AG, Frijns JH, Cornelisse CJ, et al: Does intervention improve the natural course of glomus tumors? A series of 108 patients seen in a 32-year period. Ann Otol Rhinol Laryngol 101:635-642, 1992

51. Wang ML, Hussey DH, Doornbos JF, et al: Chemodectoma of the temporal bone: a comparison of surgical and radiotherapeutic results. Int J Radiat Oncol Biol Phys 14:643-648, 1988

52. Watkins LD, Mendoza N, Cheesman AD, et al: Glomus jugulare tumours: a review of 61 cases. Acta Neurochir 130:66-70, 1994
53. Whitfield PC, Grey P, Hardy DG, et al: The surgical management of patients with glomus tumours of the skull base. $\mathbf{B r} \mathbf{~ J}$ Neurosurg 10:343-350, 1996

54. Woods CI, Strasnick B, Jackson CG: Surgery for glomus tumors: the Otology Group experience. Laryngoscope 103 (Suppl 60):65-70, 1993

Manuscript received June 10, 2004.

Accepted in final form July 7, 2004.

Address reprint requests to: William T. Couldwell, M.D., Ph.D., Department of Neurosurgery, University of Utah, 30 North 1900 E, Suite 3B409, Salt Lake City, Utah 84132. email: william.couldwell @hsc.utah.edu. 